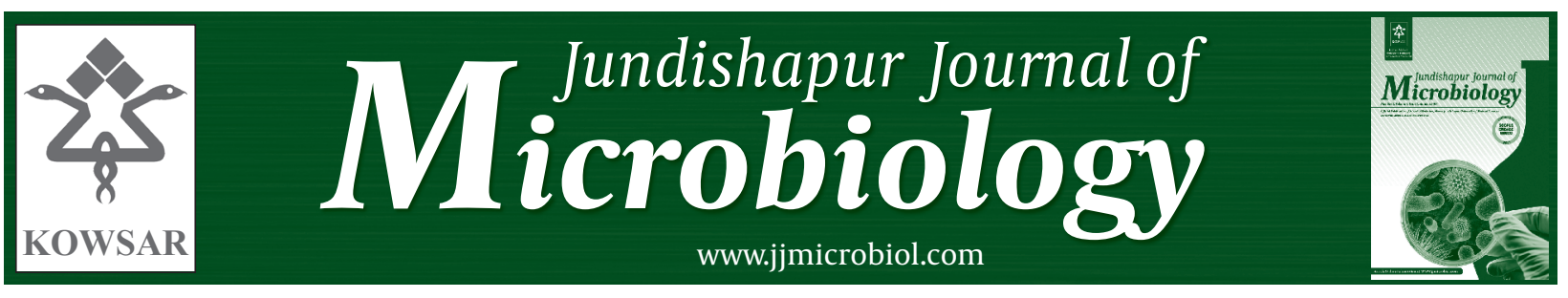

\title{
Inhibition and Disruption Properties of Chlorhexidine Gluconate on Single and Multispecies Oral Biofilms
}

\author{
Gulhas Solmaz ${ }^{1}$, May Korachi ${ }^{1^{*}}$ \\ ${ }^{1}$ Genetics and Bioengineering Department, Faculty of Engineering and Architecture, Yeditepe University Kayisdagi, Istanbul, Turkey
}

\begin{tabular}{l}
\hline A R T I C L E I N F O \\
\hline Article type: \\
Original Article \\
\hline Article history: \\
Received: 19 Mar 2012 \\
Revised: 07 May 2012 \\
Accepted: 15 May 2012 \\
\hline
\end{tabular}

Keywords:

Biofilms

Anti-microbial Agent

Chlorhexidine Gluconate

Periodontitis

\begin{abstract}
A B S T R A C T
Background: Chlorhexidine gluconate (CHX) is the most common antimicrobial agent used against oral pathogens, however information on its ability to inhibit and disrupt anaerobic single and multispecies biofilms is relatively unknown.

objectives: The purpose of this study was to test the efficacy of CHX for its biofilm inhibition and disruption properties using crystal violet assay.

Materials and Methods: Biofilm assays were carried out on single and multispecies of four oral pathogens: Streptococcus mutans, Fusobacterium nucleatum, Aggregatibacter actinomycetemcomitans and Porphyromonas gingivalis.

Results: Streptococcus mutans, Fusobacterium nucleatum and multi-species biofilm formation were inhibited in more than $90 \%$ of cases at concentrations of 3-12 mg/L. CHX exhibited strong disruptive activity ( $>65 \%$ ) on one-day old biofilms of A. actinomycetemcomitans and $P$. gingivalis.

Conclusions: In conclusion, CHX was a high effective biofilm inhibitor on S. mutans, F. nucleatum and multispecies biofilms but had a minimal effect on $P$. gingivalis and A. actinomycetemcomitans. Conversely, CHX showed disruptive properties on late colonizers in single species biofilms but not on early colonizers and multispecies biofilms.
\end{abstract}

Published by Kowsar Corp, 2013. cc 3.0.

Implication for health policy/practice/research/medical education:

This study provides practical information for both patients and dental practitioners on the efficacy of widely used antimicrobial mouthwash CHX, against oral biofilms.

- Please cite this paper as:

Solmaz G, Korachi M. Inhibition and Disruption Properties of Chlorhexidine Gluconate on Single and Multispecies Oral Biofilms. Jundishapur J.Microbiol. 2013; 6(1): 61-6. DOI: 10.5812/jjm.4852

\section{Background}

Bacteria in the oral cavity colonize in the form of communities known as dental biofilms or plaques. Dental plaque is defined as "a biofilm community that accumulates through sequential and ordered colonization of multiple oral bacteria" (1). This bacterial community is composed of bacterial micro-colonies, an extracellular slime matrix, fluid channels, and complex communication systems (2). The behavior of biofilm associated bacteria towards host and en- vironmental conditions is different than their planktonic counterparts (3). Therefore, host factors as well as biofilm growth determines the state of oral health. Shifts in composition of the predominant species in the biofilm disturbs the balance with host and may initiate oral disease (3).

To date, it is known that almost all oral diseases including dental caries and periodontal disease are caused by dental biofilms. In 1998, Socransky and co-workers grouped bacterial species in dental plaque into six colour coded bacterial complexes which determine the situation of biofilm

\footnotetext{
${ }^{*}$ Corresponding author: May Korachi, Yeditepe University, Genetics and Bioengineering Department, Faculty of Engineering and Architecture, Yeditepe University, Kayisdagi, Istanbul, Turkey. Tel:+90-2165782653, Fax:+90-2165780829, E-mail: mkorachi@yeditepe.edu.tr

DOI:10.5812/jjm.4852

(C) 2013 Ahvaz Jundishapur University of Medical Sciences; Published by Kowsar Corp.

This is an Open Access article distributed under the terms of the Creative Commons Attribution License (http://creativecommons.org/licenses/by/3.0), which permits unrestricted use, distribution, and reproduction in any medium, provided the original work is properly cited.
} 
(healthy versus diseased) (4). The presence of orange (Fusobacterium nucleatum, P. intermedia, Prevotella nigrescens) and red complex (Porphyromonas gingivalis, Tannerella forsythensis and Treponemes denticola) bacteria and a green complex bacteria, Aggregatibacter actinomycetemcomitans, in subgingival plaque have been associated with periodontitis (5). Since the presence of high number of pathogenic oral bacteria in dental biofilms is the main etiological factor for developing oral diseases (6), there is an increasing interest in the use of antimicrobial agents which can control the biofilm, by either inhibition or disruption.

Chlorhexidine Gluconate (CHX), a cationic bisbiguanide compound with high antibacterial activity, is one of the most commonly used chemotherapeutic agents against oral diseases. This is because of its wide spectrum of activity against yeasts, Gram positive, and Gram negative bacteria including many anaerobic pathogens (7). The mechanism of action is due to its cationic nature which binds to anionic compounds on the bacterial surface such as phosphate groups of teichoic acid in Gram positive and lipopolysaccharide in Gram negative bacteria and disrupts bacterial integrity (8). This leads to leakage of the cell constituents and ultimately cell death (9).

The significant effects of chlorhexidine on plaque and gingivitis have been well documented (10-12) and the effects were attributed to the reduction of pellicle formation and attachment of bacteria on the tooth surface (13). However, prolonged use of chlorhexidine carries with it several side effects including teeth staining, disturbances in taste sensation and increased calculus accumulation $(14,15)$. Several studies have previously tested the effects of chlorhexidine gluconate on oral biofilms developed on different substrata including the tooth (16), hydroxyapatite discs (17), and cellulose nitrate membrane (18). These models required the use of expensive microscopic devices for quantification of biofilms.

\section{Objectives}

The purpose of this study was to evaluate effectiveness of chlorhexidine gluconate against single and multispecies oral biofilm formation and disruption capability against early developed oral biofilms using a simple and inexpensive crystal violet assay.

\section{Materials and Methods}

\subsection{Bacterial Strains and Growth Conditions}

Bacterial strains studied were P. gingivalis (ATCC 33277), F. nucleatum (ATCC 25586), A. actinomycetemcomitans (FDC Y4), and S. mutans. All ATCC and FDC strains were kindly donated by Dr. Philip Bird, the University of Queensland, Australia. S. mutans was obtained from Yeditepe University Culture Collection. Brain Heart Broth (BHB; Merck, Germany) was used for the growth and maintenance of microorganisms while BHB supplemented by $1 \%$ sucrose was used for the growth of bacterial biofilms. All bacteria were cultured and incubated anaerobically at $37{ }^{\circ} \mathrm{C}\left(\mathrm{N}_{2}\right.$ $80 \%: \mathrm{H}_{2} 10 \%: \mathrm{CO}_{2} 10 \% \mathrm{v} / \mathrm{v}$ ) in an anaerobic work station (Don Whitley, UK) for 24 hours. Bacterial suspensions used for antibacterial assays and quantitative biofilm assays were prepared by harvesting overnight cultures, centrifugation at $2400 \mathrm{x} g$ for five minutes, re-suspending in $10 \mathrm{mM}$ phosphate buffered saline (PBS, pH 7.2), and adjusting the turbidity to 1.0 Mc Farland standard. The multi-species suspension was prepared from equal volumes from a single stock suspension of S. mutans, F. nucleatum, A. actinomycetemcomitans and $P$. gingivalis giving a final concentration of approximately 3 x $108 \mathrm{cfu} / \mathrm{mL}$ bacteria cell density, similar to the single stock densities.

\subsection{Preparation of Antimicrobial Agent}

Aqueous chlorhexidine gluconate (CHX) a commercially available mouthwash (Drogsan, Turkey) was diluted with BHB to obtain a stock solution equal to $24 \mathrm{mg} / \mathrm{L}$ in order to be used in assays performed by microdilution method.

\subsection{Antimicrobial Assay Using the Disc Diffusion Meth- od}

The disc diffusion assay was carried out according to Clinical and Laboratory Standards Institute (CLSI) (19). Briefly, prepared bacterial stock suspensions were cultured onto 5\% Sheep Blood Agar (Salubris, USA). Filter paper discs (6 mm) were impregnated with $15 \mu \mathrm{L}$ CHX and placed onto the agar. Following anaerobic incubation at $37{ }^{\circ} \mathrm{C}$ for 24 hours, the diameters of inhibition zones were measured in millimeters. The assay was repeated three times and the antibacterial activity was expressed as mean \pm standard deviations of inhibition diameters.

\subsection{MIC and MBC Determination of Chlorhexidine Glu- conate}

The Minimum Inhibitory Concentration (MIC) and Minimum Bactericidal Concentration (MBC) assays were performed using the broth microdilution method as described by Wei et al. (20), with some modifications. The MIC was defined as the lowest concentration of shiitake oil at which no visible growth (O.D. $595 \mathrm{~nm}<0.05$ ) was detected. The lowest concentration at which the original growth was reduced by $\geq 99.9 \%$ was defined as MBC.

Two fold serial dilutions of CHX with BHB medium were aliquoted into wells. Final concentrations of chlorhexidine ranged from 0.09-12 mg/L. Each well was then inoculated with $100 \mu \mathrm{L}$ of bacterial cell suspension, at a final concentration of 1.5 x $105 \mathrm{cfu} / \mathrm{mL}$ for all species. Negative control (medium and inoculum) and blank (medium and CHX) wells were included. After incubation, the absorbance was measured at $595 \mathrm{~nm}$ by a microplate reader (ThermoLab Systems, Germany) to quantify cell growth. To measure MBC, $5 \mu \mathrm{L}$ of inoculum was incubated anaerobically on 5\% sheep blood agar for 24-36 hours and the 
growth on agar surface was examined.

\subsection{Inhibition Properties of Chlorhexidine Gluconate on Biofilm Formation}

The effect of CHX on biofilm formation was determined by microdilution as well as crystal violet staining methods (19). As previously described, CHX dilutions were prepared and wells inoculated with $100 \mu \mathrm{L}$ of bacterial solution (1.5 X $105 \mathrm{cfu} / \mathrm{mL}$ ). Growth was confirmed at $595 \mathrm{~nm}$ absorbancy following incubation. Planktonic cells were removed and remaining cells were washed by PBS ( $\mathrm{pH}$ 7.2). Biofilms were fixed with methanol for 15 minutes, air dried, and stained with crystal violet (Sigma, Germany), (150 $\mu \mathrm{L}$ of $0.1 \%(w / v))$ for 15 minutes. Wells were then thoroughly washed by sterile distilled water. Biofilm formation was quantified by adding $200 \mu \mathrm{L}$ of $95 \%$ ethanol into each well. Plates were rocked for 30 minutes at room temperature and absorbance values determined for each well at O.D. 595.

The percentage of biofilm formation in the presence of different concentrations of chlorhexidine gluconate was determined using the equation:(O.D. 595 of the test well / O.D. 595 of non-treated control well) $x 100$.

\subsection{Disruption Properties of Chlorhexidine Gluconate on Early Developed Biofilms}

To examine the effect of chlorhexidine gluconate on the one-day developed biofilms, plates were prepared as for inhibition assay by exclusion of the addition of CHX, and anaerobically incubated for 24 hours. As before, cells were washed and CHX dilutions $(0.09-12 \mathrm{mg} / \mathrm{L})$ were aliquoted into the wells. The microplate was then incubated for a further 24 hours and biofilms quantified as before.

\subsection{Statistical Analysis}

Data were analyzed for the mean and standard deviation of at least two independent tests with duplicates for each (SPSS version 10).

\section{Results}

\subsection{Antimicrobial Assay}

Results for three independent antimicrobial tests are shown in Table 1 as diameters of inhibition zones and standard deviations. CHX exhibited significant antimicrobial activity on all test microorganisms showing inhibition diameters greater than $10 \mathrm{~mm}$. Results of the disc diffusion assays indicated that $\mathrm{CHX}$ worked the best against Fusobacterium followed by P. gingivalis, A. actinomycetemcomitans, and S. mutans.

\subsection{MIC and $M B C$}

The concentration of CHX required to inhibit (MIC) and to kill (MBC) planktonic bacteria are shown in Table 1. Among the bacterial species tested, S. mutans was observed to be the most susceptible to CHX MIC $(1.5 \mathrm{mg} / \mathrm{L})$ followed by A. actinomycetemcomitans, F. nucleatum, and $P$. gingivalis, respectively. All MICs corresponded with their MBC values indicating bactericidal activity of the test agent, with the exception of S. mutans and A. actinomycetemcomitans where 2-fold higher MBC values were detected. Furthermore, CHX exhibited inhibitory and cidal effects at a concentration of $6 \mathrm{mg} / \mathrm{L}$ on the mixed culture.

\subsection{Biofilm Inhibition}

The inhibitory effects of CHX on biofilm formation were tested by microdilution broth method for single and mixed cultures. Generally, an agent is assumed to be a specific biofilm inhibitor if it is effective on biofilm formation at doses lower than its MIC, since at these doses biofilm formation might be inhibited by a different mode of action rather than killing the bacteria (21). CHX acted as specific biofilm inhibitor on F. nucleatum, since inhibition occurred at sub-inhibitory concentration ( $3 \mathrm{mg} / \mathrm{L}$ ) (Figure 1). Furthermore, CHX at concentrations from 3 to $12 \mathrm{mg} / \mathrm{L}$ exhibited a strong inhibition activity ( $>95 \%$ ) on formation of S. mutans and F. nucleatum biofilms. While P. gingivalis biofilms were inhibited more than $40 \%$ (6-12 $\mathrm{mg} / \mathrm{L}$ ), A actinomycetemcomitans was not significantly affected. Multi-species biofilm formation was also examined in the presence of CHX. More than $90 \%$ of the biofilm was inhibited even at its sub-MIC concentrations (1.5 and $3 \mathrm{mg} / \mathrm{L})$.

\subsection{Biofilm Disruption}

It is known that once established, biofilms have greater resistance to external agents such as antibiotics, deter-

\begin{tabular}{|c|c|c|c|}
\hline & Diameter of Inhibition Zone, mma $^{a}$ & MIC, $\mathbf{m g} / \mathrm{L}$ & MBC,mg/L \\
\hline S. mutans & $15.8 \pm 3.4$ & 1.5 & $3 \uparrow^{b}$ \\
\hline A. actinomycetemcomitans & $19.2 \pm 2.8$ & 3 & $6 \uparrow^{b}$ \\
\hline F. nucleatum & $25.3 \pm 0.1$ & 6 & 6 \\
\hline P. gingivalis & $21.2 \pm 4$ & 6 & 6 \\
\hline Mix bacteria & $\mathrm{NA}^{\mathrm{c}}$ & 6 & 6 \\
\hline
\end{tabular}

a Each value is mean \pm standard deviation. Each experiment was carried out three times.

${ }^{\mathrm{b}} \uparrow$, indicates the essential oil having a MBC value higher than its MIC value.

"NA, indicates that this test was "non-applicable". This test was not applied for mix bacteria. 


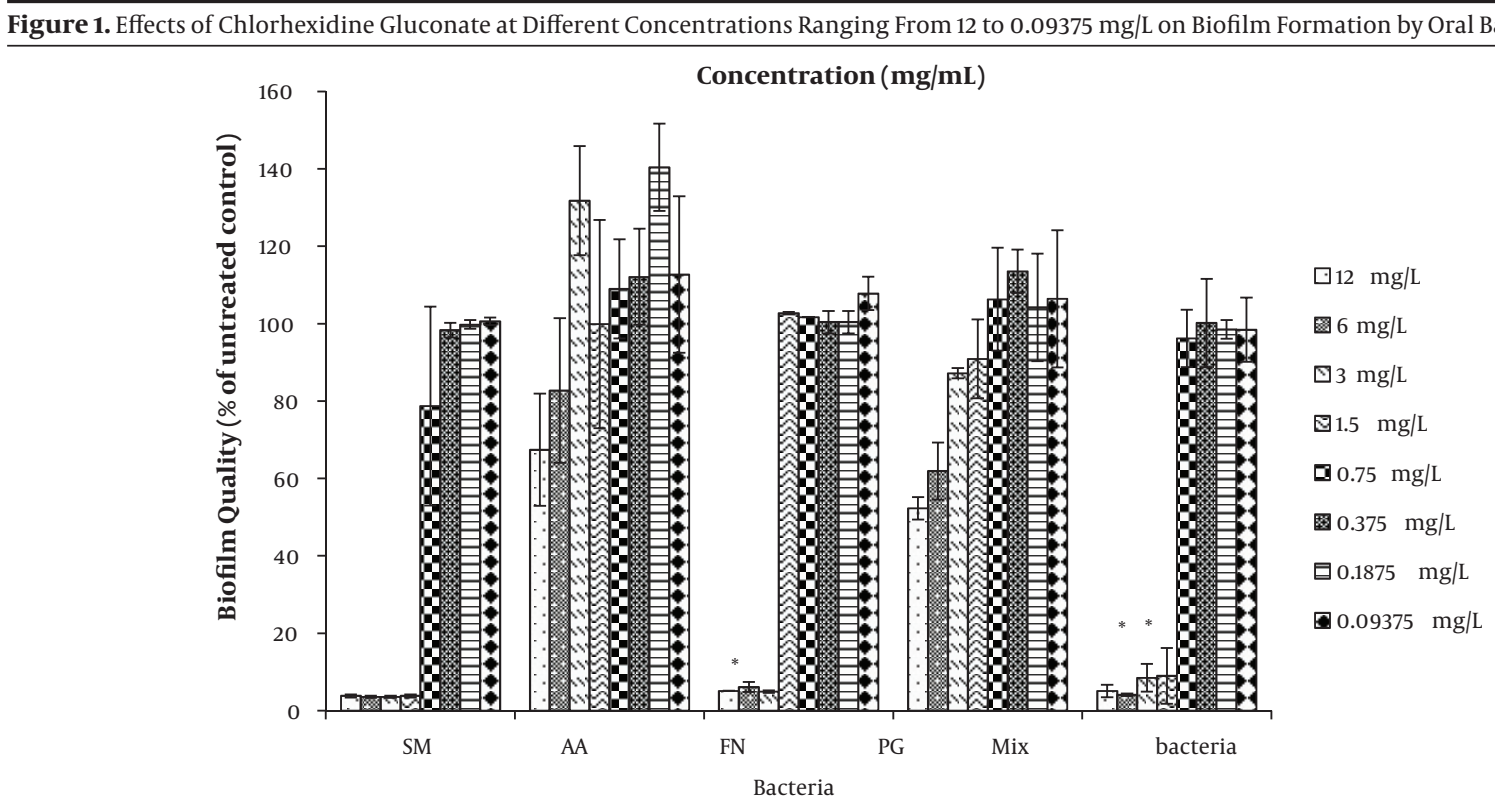

Data represent the mean and standard deviation of at least two independent tests with duplicates for each. Asterisk shows the percentage of biofilm formation $<60 \%$ at sub-inhibitory concentrations (sub-MIC) of chlorhexidine gluconate. SM: S. mutans, AA: A. actinomycetemcomitans, FN: F. nucleatum, PG: P. gingivalis, and Mix: multi-species biofilms.

Figure 2. Effects of Chlorhexidine Gluconate at Different Concentrations Ranging From 12 to $0.09375 \mathrm{mg} / \mathrm{L}$ on One-Day Developed Biofilm Formed by Oral Bacteria

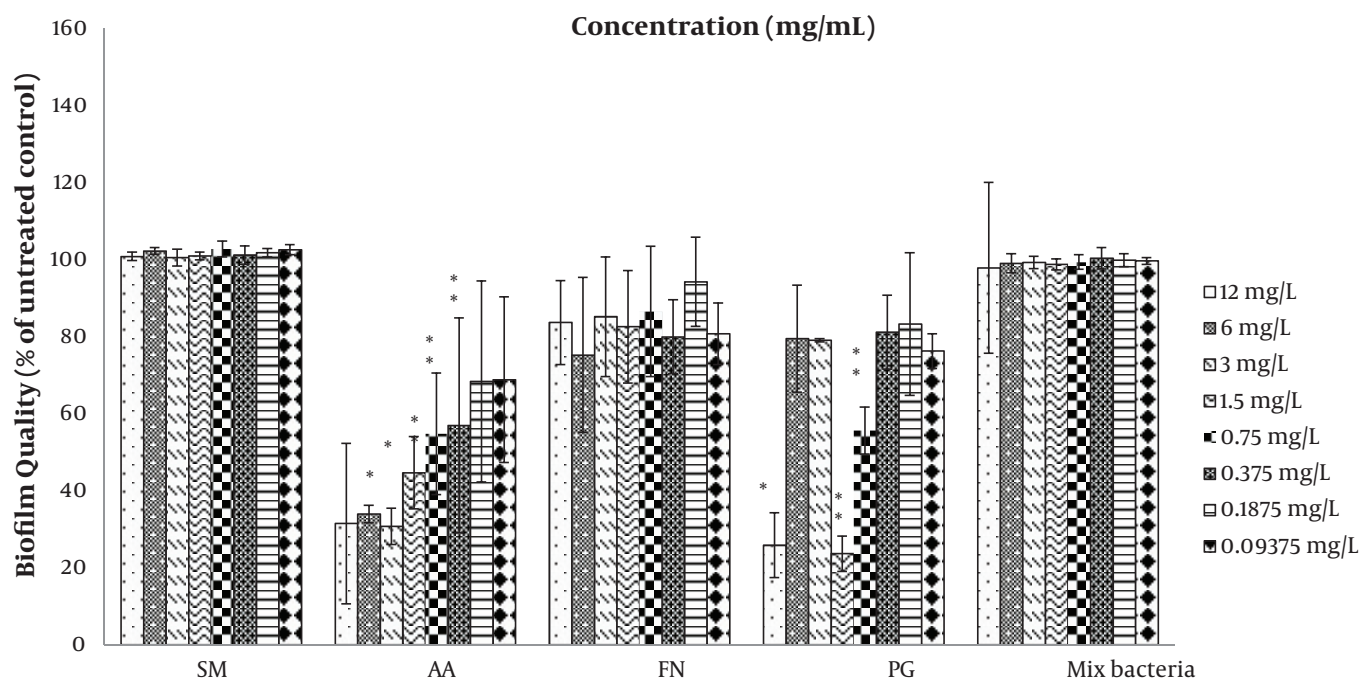

Data represent the mean and standard deviation of at least two independent tests with duplicates for each. Asterisk shows the percentage of biofilm quantity $<60 \%$ at $2 \mathrm{X}$ or $1 \mathrm{X}$ inhibitory concentrations (MIC) of chlorhexidine gluconate. Double asterisks show the percentage of biofilm quantity $<60 \%$ at sub-inhibitory concentrations (sub-MIC) of chlorhexidine gluconate. SM: S. mutans, AA:A. actinomycetemcomitans, FN: F. nucleatum, PG: P. gingivalis and Mix: multi-species biofilms.

gents, or biocides than their planktonic cells (22) and therefore disruption of pre-formed biofilms tend to have higher MIC values than killing of planktonic cells in suspension (23). By considering this fact, disruptive effects at MIC and 2X MIC values of CHX were also assumed to be efficient.

Results (Figure 2) showed that chlorhexidine gluconate reduced one-day developed biofilms of A. actinomycetemcomitans and $P$. gingivalis more than $65 \%$ at their $2 \mathrm{X}$ and $4 \mathrm{X}$ MIC values when compared to the controls. This agent at its sub-MIC concentration was effective but to a lesser extent on preformed biofilms of A. actinomycetemcomitans and $P$. gingivalis. Early developed biofilms of $F$. nucleatum and $S$. mutans exhibited resistance to all test concentra- 
tions of CHX. Moreover, multi-species biofilms were seen to be resistant to CHX as even at its supra-MIC values.

\section{Discussion}

The antimicrobial activity of CHX on planktonic species of oral pathogens was evaluated by disc diffusion, MIC, and MBC assays. Although CHX exhibited antibacterial activity against all test bacteria, MIC results revealed CHX inhibited growth at concentrations lower than undiluted agent. However, these MIC results did not correlate with that of disc diffusion test, i.e., the statement "when zone of growth inhibition decreases, MIC increases" (24) was not observed in all cases. The correlation between disc diffusion and MIC results has been previously discussed and some studies have shown that these tests do not always correlate $(25,26)$. Previous studies have shown similar results to our MIC findings (27). Furthermore, MIC findings observed in this study are in correlation with the general statement that Gram positive bacteria are more susceptible to chlorhexidine than Gram negative bacteria (28).

S. mutans, the only Gram positive bacteria tested, was affected the most by chlorhexidine gluconate. Several biofilm models using different kinds of substratum have been used in previous studies including tooth, hydroxyapatite discs, and cellulose nitrate membrane $(17,18,29)$. However, these bear a disadvantage as they can be timeconsuming, The use of crystal violet assay in this study reveals several advantages including allowing a large number of variables to be tested quickly and easily, and indirect quantification of biofilms formed on both the bottom and sides of the well by a very simple staining step (30). Moreover, in a study of Luppens and Ten Cate (31), it has been shown that S. mutans biofilm development on polystyrene surface was similar to that on hydroxyapatite in terms of viability and protein expression. Therefore, this was chosen as the biofilm model for this study.

Results of biofilm inhibition assay have shown that biofilm formation of S. mutans, F. nucleatum, and multiple bacteria were more susceptible to $\mathrm{CHX}$, followed by that of $P$. gingivalis and A. actinomycetemcomitans. Moreover, there was a dose-dependent response effect of chlorhexidine (12-1.5 mg/L) toward the biofilm formation of $S$. $m u$ tans, F. nucleatum, and mix bacteria (Figure 1). These results are comparable with previous studies in which CHX at concentrations $5 \mathrm{mg} / \mathrm{L}$ and $0.01 \mu \mathrm{L} / \mathrm{mL}(\sim 10 \mathrm{mg} / \mathrm{L})$ were shown to inhibit biofilm formation of S. mutans (32) and F. nucleatum (33), respectively. Another study on S. mutans, Streptococcus sanguis, and Actinomyces viscous (34) revealed the biofilms to only be inhibited using high concentrations of CHX (16-40 mg/L). This is most likely due to the different composition of multi-species biofilms. No disruptive capability of CHX on one-day biofilms of multi-species was observed even at its supra-MIC values.
This correlates with previous findings that have shown a disruptive effect on six-hours old biofilms but not on 24 and 48 hours-old mature multi-species biofilms (35). In another study (36), it was also shown that 0.12 percent chlorhexidine gluconate mouth-rinse exhibited little antiplaque and antigingivitis effects on mature biofilms.

In conclusion, the crystal violet assay is an easy, quick, and effective way for studying anti- biofilm agents. CHX was a highly effective biofilm inhibitor on S. mutans, F. nucleatum, and multispecies biofilms but exhibited a limited effect on P. gingivalis and A. actinomycetemcomitans. Conversely, CHX showed disruptive properties on late colonizers in single species biofilms but not on early colonizers and multispecies biofilms.

\section{Acknowledgements}

The authors would like to thank Dr. Philip Bird, University of Queensland for provision of the bacterial ATCC strains.

\section{Financial Disclosure}

None Declared.

\section{Funding/Support}

This research was supported by Yeditepe University.

\section{Authors' Contribution}

None Declared.

\section{References}

1. Hojo K, Nagaoka S, Ohshima T, Maeda N. Bacterial interactions in dental biofilm development. J Dent Res. 2009;88(11):982-90.

2. Marsh P. Dental plaque as a microbial biofilm. Caries Res. 2004;38(3):204-11.

3. Sbordone L, Bortolaia C. Oral microbial biofilms and plaque-related diseases: microbial communities and their role in the shift from oral health to disease. Clin Oral Investig. 2003;7(4):181-8.

4. Socransky SS, Haffajee AD, Cugini MA, Smith C, Kent RL, Jr. Microbial complexes in subgingival plaque. J Clin Periodontol. 1998;25(2):134-44.

5. Walker C, Sedlacek MJ. An in vitro biofilm model of subgingival plaque. Oral Microbiol Immunol. 2007;22(3):152-61.

6. Palmer RJ, Darveau R, Lamont RJ, Nyvad B, Teles RP. Human oral bacterial biofilms: composition, dynamics, and pathogenesis. In: Bjarnsholt T, Jensen P, Moser C, Høiby N, editors. Biofilm Infections. New York: Springer; 2011. p. 35-68.

7. Young MP, Korachi M, Carter DH, Worthington HV, McCord JF, Drucker DB. The effects of an immediately pre-surgical chlorhexidine oral rinse on the bacterial contaminants of bone debris collected during dental implant surgery. Clin Oral Implants Res. 2002;13(1):20-9.

8. Estrela C, Ribeiro RG, Estrela CR, Pecora JD, Sousa-Neto MD. An timicrobial effect of $2 \%$ sodium hypochlorite and $2 \%$ chlorhexidine tested by different methods. Braz Dent J. 2003;14(1):58-62.

9. Siqueira JF, Jr., Rocas IN, Paiva SS, Guimaraes-Pinto T, Magalhaes KM, Lima KC. Bacteriologic investigation of the effects of sodium hypochlorite and chlorhexidine during the endodontic treatment of teeth with apical periodontitis. Oral Surg Oral Med Oral Pathol Oral Radiol Endod. 2007;104(1):122-30.

10. Lorenz K, Bruhn G, Heumann C, Netuschil L, Brecx M, Hoffmann 
T. Effect of two new chlorhexidine mouthrinses on the development of dental plaque, gingivitis, and discolouration. A randomized, investigator-blind, placebo-controlled, 3-week experimental gingivitis study.J Clin Periodontol. 2006;33(8):561-7.

11. Nayak PA, Nayak UA, Mythili R. Effect of Manuka honey, chlorhexidine gluconate and xylitol on the clinical levels of dental plaque. Contemp Clin Dent. 2010;1(4):214-7.

12. Sandhu M, Grewal N. Clinical Effectiveness of Chlorhexidine and Sodium Fluoride Mouth Rinses on Plaque and Gingivitis in Mentally Disabled Children and Normal Children. JIDA. 2011;5(1):081.

13. Fiorellini JP, Paquette DW. The potential role of controlled-release delivery systems for chemotherapeutic agents in periodontics. Curr Opin Dent. 1992;2:63-79.

14. McCoy LC, Wehler CJ, Rich SE, Garcia RI, Miller DR, Jones JA. Adverse events associated with chlorhexidine use: results from the Department of Veterans Affairs Dental Diabetes Study.J Am Dent Assoc. 2008;139(2):178-83.

15. Turkoglu O, Becerik S, Emingil G, Kutukculer N, Baylas H, Atilla G. The effect of adjunctive chlorhexidine mouthrinse on clinical parameters and gingival crevicular fluid cytokine levels in untreated plaque-associated gingivitis. Inflamm Res. 2009;58(5):27783.

16. Lundstrom JR, Williamson AE, Villhauer AL, Dawson DV, Drake DR. Bactericidal activity of stabilized chlorine dioxide as an endodontic irrigant in a polymicrobial biofilm tooth model system. JEndod. 2010;36(11):1874-8.

17. Noiri Y, Okami Y, Narimatsu M, Takahashi Y, Kawahara T, Ebisu S. Effects of chlorhexidine, minocycline, and metronidazole on Porphyromonas gingivalis strain 381 in biofilms. J Periodontol. 2003;74(11):1647-51.

18. Sena NT, Gomes BP, Vianna ME, Berber VB, Zaia AA, Ferraz CC, et al. In vitro antimicrobial activity of sodium hypochlorite and chlorhexidine against selected single-species biofilms. Int Endod J. 2006;39(11):878-85.

19. Watts JL, Clinical, Institute LS. Performance standards for antimicrobial disk and dilution susceptibility tests for bacteria isolated from animals: approved standard. Clinical and Laboratory Standards Institute (CLSI); 2008.

20. Wei GX, Campagna AN, Bobek LA. Effect of MUC7 peptides on the growth of bacteria and on Streptococcus mutans biofilm. J Antimicrob Chemother. 2006;57(6):1100-9.

21. Marsh PD. Microbiological aspects of the chemical control of plaque and gingivitis. J Dent Res. 1992;71(7):1431-8.

22. Sandasi M, Leonard C, Viljoen A. The effect of five common essential oil components on Listeria monocytogenes biofilms. Food control. 2008;19(11):1070-5.

23. Filoche SK, Soma K, Sissons CH. Antimicrobial effects of essential oils in combination with chlorhexidine digluconate. Oral Microbiol Immunol. 2005;20(4):221-5.

24. McClatchey KD. Clinical laboratory medicine. Lippincott Williams
\& Wilkins; 2002.

25. Hajia M, Qorbanalizadehgan M, Rahbar M, Izadi M. Laboratory evaluation of Iranian commercially provided antibiotic disks with conventional e-test method for susceptibility testing in three most isolated multi-drug resistant organisms. Journal [serial on the Internet]. 2011 Date; 5(1): Available from: http:|| www.ispub.com/journal/the-internet-journal-of-microbiology/ volume-5-number-1/laboratory-evaluation-of-iranian-commercially-provided-antibiotic-disks-with-conventional-e-test-method-for-susceptibility-testing-in-three-most-isolated-multi-drug resistant-organisms.html.

26. Klement E, Chaffer M, Leitner G, Shwimmer A, Friedman S, Saran A, et al. Assessment of accuracy of disk diffusion tests for the determination of antimicrobial susceptibility of common bovine mastitis pathogens: a novel approach. Microbial Drug Resistance. 2005;11(4):342-50.

27. Greenberg M, Urnezis P, Tian M. Compressed mints and chewing gum containing magnolia bark extract are effective against bacteria responsible for oral malodor. J Agric Food Chem. 2007;55(23):9465-9.

28. Koljalg S, Naaber P, Mikelsaar M. Antibiotic resistance as an indicator of bacterial chlorhexidine susceptibility. J Hosp Infect. 2002;51(2):106-13.

29. Spratt DA, Pratten J, Wilson M, Gulabivala K. An in vitro evaluation of the antimicrobial efficacy of irrigants on biofilms of root canal isolates. Int Endod J. 2001;34(4):300-7.

30. Froeliger EH, Fives-Taylor P. Streptococcus parasanguis fimbriaassociated adhesin fap 1 is required for biofilm formation. Infect Immun. 2001;69(4):2512-9.

31. Luppens SB, ten Cate JM. Effect of biofilm model, mode of growth, and strain on streptococcusmutans protein expression as determined by two-dimensional difference gel electrophoresis. J Proteome Res. 2005;4(2):232-7.

32. Altman H, Steinberg D, Porat Y, Mor A, Fridman D, Friedman M, et al. In vitro assessment of antimicrobial peptides as potential agents against several oral bacteria.J Antimicrob Chemother. 2006;58(1):198-201.

33. Gursoy UK, Gursoy M, Gursoy OV, Cakmakci L, Kononen E, Uitto VJ. Anti-biofilm properties of Satureja hortensis L. essential oil against periodontal pathogens. Anaerobe. 2009;15(4):164-7.

34. Yanti, Rukayadi Y, Lee KH, Hwang JK. Activity of panduratin A isolated from Kaempferia pandurata Roxb. against multi-species oral biofilms in vitro. J Oral Sci. 2009;51(1):87-95.

35. Zaura-Arite E, Van Marle J, Ten Cate J. Confocal microscopy study of undisturbed and chlorhexidine-treated dental biofilm. J Dent Res. 2001;80(5):1436-40.

36. Zanatta FB, Antoniazzi RP, Rosing CK. The effect of $0.12 \%$ chlorhexidine gluconate rinsing on previously plaque-free and plaquecovered surfaces: a randomized, controlled clinical trial. J Periodontol. 2007;78(11):2127-34. 